\title{
FUNCTIONS WITH A UNIQUE MEAN VALUE AND AMENABILITY
}

\author{
YUJI TAKAHASHI
}

(Communicated by J. Marshall Ash)

\begin{abstract}
It is shown that there exist many amenable locally compact groups for which the sets of functions with unique left invariant mean values are not closed under addition. This resolves negatively a problem raised by $\mathrm{T}$. Miao.
\end{abstract}

Let $G$ be a locally compact group with a fixed left Haar measure $\lambda$ and let $L^{p}(G) \quad(1 \leq p \leq \infty)$ be the associated Lebesgue spaces. (As usual, if $G$ is compact, then $\lambda$ is assumed to be normalized, i.e., $\lambda(G)=1$.) A subspace $S$ of $L^{\infty}(G)$ is said to be admissible if it contains the constants and ${ }_{x} f$ for each $f \in S$ and $x \in G$ (where ${ }_{x} f$ is the left translate of $f$ by $x,{ }_{x} f(y)=f(x y)$ $(y \in G)$ ). If $f \in L^{\infty}(G)$, let $S_{f}$ denote the smallest admissible subspace containing $f$. We say that $f \in L^{\infty}(G)$ has a unique left invariant mean value if $\operatorname{LIM}\left(S_{f}\right)$ is nonempty and there exists a constant $c$ such that $m(f)=c$ for each $m \in \operatorname{LIM}\left(S_{f}\right)$. (For an admissible subspace $S$ of $L^{\infty}(G), \operatorname{LIM}(S)$ stands for the set of left invariant means on $S$, i.e., all $m \in S^{*}$ with $m \geq 0$, $m(1)=1$, and $m\left({ }_{x} f\right)=m(f) \quad(x \in G, f \in S)$.) The set of functions with a unique left invariant mean value is denoted by $U(G)$. Note that $U(G)$ is always closed under scalar multiplication. Recall also that if $G$ is amenable as a discrete group, then $U(G)$ coincides with the sum of the constants and the norm closed linear span of $\left\{f-{ }_{x} f: f \in L^{\infty}(G), x \in G\right\}$ (see [6, Theorem 1.1]). In particular, $U(G)$ is closed under addition for such a $G$. Recently Miao [3, Theorem 3.4] proved that if $U(G)$ is closed under addition, then $G$ is amenable, thus answering a question raised by Rosenblatt and Yang in [6, $\mathrm{p}$. 747]. The following was posed as an open problem in [3, p. 1083]: Is $U(G)$ closed under addition if $G$ is amenable? The purpose of the present note is to give many examples which show that the answer to Miao's problem is negative.

Recall that a compact group $G$ is said to have the mean zero weak containment property if there exists a net $\left\{g_{\alpha}\right\}$ in

$$
L_{0}^{2}(G)=\left\{f \in L^{2}(G): \int_{G} f d \lambda=0\right\}
$$

Received by the editors October 9, 1992.

1991 Mathematics Subject Classification. Primary 43A07, 43A15, 22 C05.

Key words and phrases. Locally compact groups, amenable groups, functions with a unique left invariant mean value, mean zero weak containment property. 
such that $\left\|g_{\alpha}\right\|_{2}=1$ for all $\alpha$ and $\lim _{\alpha}\left\|_{x} g_{\alpha}-g_{\alpha}\right\|_{2}=0$ for all $x \in G$ (see [5]). The negative answer to Miao's problem is a direct consequence of the following result.

Theorem. Let $G$ be an infinite compact group and suppose that $U(G)$ is closed under addition. Then $G$ has the mean zero weak containment property.

Proof. Assume, by way of contradiction, that $G$ does not have the mean zero weak containment property. We denote by $\mathbb{C}$ and $H$ the constant functions on $G$ and the linear span of $\left\{f-{ }_{x} f: f \in L^{\infty}(G), x \in G\right\}$, respectively. Let us first show that $H+\mathbb{C}$ is included in $U(G)$. Obviously, $\mathbb{C}$ is contained in $U(G)$. Let $h$ be in $\left\{f-{ }_{x} f: f \in L^{\infty}(G), x \in G\right\}$. Since $\lambda$ induces a left invariant mean on $S_{h}, \operatorname{LIM}\left(S_{h}\right)$ is nonempty. Observe that

$$
\lim _{n \rightarrow \infty}\left\|\frac{1}{n} \sum_{k=1}^{n}{ }_{x^{k}}\left(f-{ }_{x} f\right)\right\|_{\infty}=0
$$

for each $f \in L^{\infty}(G)$ and $x \in G$. Using this fact, we can see that $m(h)=0$ for each $m \in \operatorname{LIM}\left(S_{h}\right)$. Thus $h$ has a unique left invariant mean value. As $U(G)$ is closed under addition, $U(G)$ contains $H+\mathbb{C}$, as desired. On the other hand, since $G$ does not have the mean zero weak containment property, it follows from the proofs of [8, Lemma and Proposition 2] that $L^{\infty}(G)=H+\mathbb{C}$. In conclusion, we obtain $L^{\infty}(G)=U(G)$. But this is a contradiction. In fact, let $E$ be an open dense subset of $G$ satisfying $\lambda(E)<1$. (It is possible to find such an $E$ because $G$ is an infinite compact group.) Then there exists a left invariant mean $m$ on $S_{\chi_{E}}$ such that $m\left(\chi_{E}\right)=1$, where $\chi_{E}$ denotes the characteristic function of $E$ (cf. [3, Lemma 3.1]). This means that both 1 and $\lambda(E) \quad(<1)$ can be left invariant mean values of $\chi_{E}$ on $S_{\chi_{E}}$. We therefore have $\chi_{E} \notin U(G)$, which gives the desired contradiction. The proof is now complete.

It is well known that if an infinite compact group $G$ is amenable as a discrete group, then $G$ has the mean zero weak containment property (cf. [4, Theorem 1.3 and Lemma 3.1]). Some examples of compact groups which do not have the mean zero weak containment property can be found in $[1,2,5,7]$. For example, $\mathrm{SO}(n)$ (the special orthogonal group) does not have the mean zero weak containment property for $n \geq 3$. Therefore our Theorem implies that the set of functions on $\mathrm{SO}(n) \quad(n \geq 3)$ with a unique left invariant mean value is not closed under addition. This resolves Miao's problem [3, p. 1083] negatively.

As a consequence of our Theorem we have the following. This gives a number of examples which show that the answer to Miao's problem is negative.

Proposition. Let $G_{1}$ be an infinite compact group that does not have the mean zero weak containment property and let $G_{2}$ be an amenable locally compact group. Then $G_{1} \times G_{2}$ is an amenable locally compact group for which $U\left(G_{1} \times G_{2}\right)$ is not closed under addition.

Proof. Let us find two functions in $U\left(G_{1} \times G_{2}\right)$ whose sum does not have a unique left invariant mean value. Since, by the Theorem, $U\left(G_{1}\right)$ is not closed under addition, there exist $h$ and $k$ in $U\left(G_{1}\right)$ such that $h+k$ is not contained in $U\left(G_{1}\right)$. Now define functions $f$ and $g$ in $L^{\infty}\left(G_{1} \times G_{2}\right)$ by

$$
f(x, y)=h(x) \text { and } g(x, y)=k(x) \quad\left((x, y) \in G_{1} \times G_{2}\right) .
$$


Then $f$ and $g$ have unique left invariant mean values but $f+g$ does not. This can be easily verified from [6, Proposition 1.3] and a straightforward argument. Thus we obtain that $U\left(G_{1} \times G_{2}\right)$ is not closed under addition.

Remarks. (1) It is possible that a compact group $G$ has the mean zero weak containment property, and $U(G)$ is not closed under addition. For example, take $G=\mathrm{SO}(n) \times \mathbb{T}(n \geq 3)$, where $\mathbb{T}$ denotes the circle group. Then the Proposition implies that $U(G)$ is not closed under addition. Since $L^{\infty}(G)$ has more than one left invariant mean, it follows from [4, Theorem 1.3 and Lemma $3.1]$ that $G$ has the mean zero weak containment property. Thus the converse of our Theorem is false.

(2) Recall Miao's Theorem [3, Theorem 3.4] quoted above: If $U(G)$ is closed under addition, then $G$ is amenable. Our Theorem and Proposition may be regarded as partial improvements of this result. It would be of interest to determine the class of locally compact groups $G$ for which $U(G)$ is closed under addition.

(3) Let $H$ be as in the proof of our Theorem. It is worthwhile to observe the following: If $G$ is a locally compact group and if $U(G)$ is closed under addition, then

$$
H+\mathbb{C} \subseteq U(G) \subseteq \bar{H}+\mathbb{C}(=\overline{H+\mathbb{C}}),
$$

where the over bar denotes the norm closure in $L^{\infty}(G)$. The argument used in the proof of the Theorem can apply to show the first inclusion relation. (Notice that the closure of $U(G)$ under addition implies amenability of $G[3$, Theorem 3.4].) The second inclusion relation follows from the proof of [3, Theorem 3.6].

\section{REFERENCES}

1. V. G. Drinfeld, Finitely additive measure on $S^{2}$ and $S^{3}$, invariant with respect to rotations, Functional Anal. Appl. 18 (1984), 245-246.

2. G. Margulis, Some remarks on invariant means, Monatsh. Math. 90 (1980), 233-235.

3. T. Miao, Amenability of locally compact groups and subspaces of $L^{\infty}(G)$, Proc. Amer. Math. Soc. 111 (1991), 1075-1084.

4. J. Rosenblatt, Uniqueness of invariant means for measure-preserving transformations, Trans. Amer. Math. Soc. 265 (1981), 623-636.

5. _ Translation-invariant linear forms on $L^{p}(G)$, Proc. Amer. Math. Soc. 94 (1985), 226-228.

6. J. Rosenblatt and Z. Yang, Functions with a unique mean value, Illinois J. Math. 34 (1990), 744-764.

7. D. Sullivan, For $n>3$ there is only one finitely additive rotationally invariant measure on the $n$-sphere defined on all Lebesgue measurable subsets, Bull. Amer. Math. Soc. (N.S.) 4 (1981), 121-123.

8. G. A. Willis, Continuity of translation invariant linear functionals on $C_{0}(G)$ for certain locally compact groups $G$, Monatsh. Math. 105 (1988), 161-164.

Department of Mathematics, Hokkaido University of Education, Hakodate, Hachiman-Cho, Hakodate, 040 Japan 